\title{
ANÁLISE ECONÔMICA DE SISTEMAS DE PRODUÇÃO COM BOVINOCULTURA DE LEITE DA DEPRESSÃ̃O CENTRAL DO RIO GRANDE DO SUL ${ }^{1}$
}

\author{
ECONOMIC ANALYSIS OF PRODUCTION SYSTEMS OF DAIRY \\ CATTLE FARMS IN CENTRAL RIO GRANDE DO SUL, BRAZIL
}

\author{
Jorge Nunes Portela ${ }^{2}$ Julio Viégas ${ }^{3}$ Pedro Selvino Neumann ${ }^{4}$ \\ Benedito Silva Neto ${ }^{5}$ Luciany Dias Laurentino ${ }^{6}$.
}

RESUMO

O presente trabalho foi desenvolvido junto às unidades de produção familiar (UPFs) associadas à Cooperativa dos Produtores de Leite de Santa Maria (COOPROL) RS, como objetivo de identificar o desempenho econômico e os sistemas de produção existentes entre as mesmas. Foram coletadas as informações de 53 propriedades, sendo identificados os seguintes sistemas de produção: a) leite e arroz com tração animal (TA) $e$ superfície agrícola útil (SAU) média de 30ha; b) leite e arroz com tração mecanizada (TM) e SAU média de 54ha; c) leite e hortigranjeiros com TA e SAU média de 20ha; d) leite e hortigranjeiros com TM e SAU média de 50ha; e) leite e bovinos de corte com TA e SAU média de 40ha; f) leite e bovinos de corte com TA e SAU média de 308ha; g) leite e bovinos de corte com TM e SAU média de 80ha; h) leite estreme com TA e SAU média de $2 \mathrm{ha}$; i) leite estreme com TM e SAU média de 45ha. Os sistemas $A, B, C, F, H$ e I apresentam um desempenho econômico por unidade de trabalho homem (UTH) superior ao salário mínimo regional, enquanto os sistemas $D, E$ e $G$ apresentam desempenho abaixo deste indicador.

Palavras-chave : unidade de produção familiar, renda agrícola, remuneração monetária da família.

\section{SUMMARY}

This work was developed in family production units (UPFs), associated to a co-operative society, the Cooperativa dos Produtores de Leite de Santa Maria (COOPROL) RS. The goal

\begin{abstract}
was to identify the economical performance and the different systems of production used by the UPFs. The information was obtained from 53 farms, and among them the following production systems were identified: a) Milk associated to rice crops, with animal traction (AT) and an average of 30ha of tillable area (TA); b) Milk associated to rice crops with mechanical traction $(M T)$ and $T A=54 h a ; c)$ Milk associated to olericulture with $A T$ and $T A=20 h a ; d)$ Milk associated to olericulture with MT and TA=50ha; e) Milk associated to beef cattle with AT and TA=40ha; f) Milk associated to beef cattle with AT and TA=308ha; g) Milk associated to beef cattle with MT and $T A=80$ ha; h) Milk with AT and TA=20ha; i) Milk with $M T$ and $T A=45$ ha. It was observed that the systems $a, b, c, f, h$ and $i$ showed an economic perform ance per worker labour unit (WLU) superior to the regional minimum wages, while the systems $d$, e, f presented a performance below the minimum wages.
\end{abstract}

Key words: family production unit, agriculture wages, family income.

\section{INTRODUÇÃO}

A atividade de produção de leite, embora tenha permitido, no decorrer da última década, a permanência de muitas famílias no meio rural, tem vivenciado momentos de estagnação e de descapitalização acentuada de um significativo

\footnotetext{
${ }^{1}$ Parte do trabalho de dissertação apresentado à Universidade Federal de Santa Maria (UFSM), pelo primeiro autor para obtenção do título de Mestre.

${ }^{2}$ Zootecnista, Mestre em Produção Animal, UFSM. E-mail: jorgenp@eafpjk.com.br.

${ }^{3}$ Doutor em Zootecnia, professor do Departamento de Zootecnia, Centro de Ciências Rurais (CCR), UFSM, 97105-900, Santa MariaRS. E-mail: jviegas@ccr.ufsm.br. Autor para correspondência.

${ }^{4}$ Doutorando em Desenvolvimento Rural, Professor do Departamento Ensino Agrícola e Extensão Rural, CCR, UFSM.

${ }^{5}$ Doutor em Desenvolvimento Agrícola, Professor do Departamento de Estudos Agrários, UNIJUÍ.

${ }^{6}$ Acadêmico do Curso de Zootecnia, CCR, UFSM.
} 
percentual de propriedades. Segundo ESCOSTEGUY et al. (1993), no RS, as propriedades leiteiras apresentam, em média, rebanho composto de 7 vacas com uma área destinada à produção de leite de 11 ha e a produção diária de $24,5 \mathrm{~kg}$ de leite, esta integrada com outras culturas e criações, sendo a explicação da sobrevivência de algumas unidades de produção familiar (UPFs).

Para TESTA et al. (1996), a atividade leiteira apresenta determinadas particularidades sendo a atividade mais adequada à produção de caráter familiar, pois apresenta alta absorção de mão-de-obra e exerce um importante papel na formação da renda dos agricultores (melhorando o fluxo de caixa), mas não deve ser especializada, para permitir menor escala de produção, facilitar a reciclagem de dejetos, maior capacidade de absorção das crises de mercado, etc.

Conforme NETO et al. (1998), as dificuldades impostas pelo fim dos subsídios na década de 80 tiveram como conseqüência uma progressiva reorientação dos sistemas de produção de grãos, na região de Três de Maio - RS, para a pecuária de leite, cujo potencial de aumento do valor agregado por unidade de área é maior. Entretanto, esta reorientação acontece num contexto de escassez e encarecimento do crédito agrícola, permitindo apenas aos agricultores que conseguem uma renda relativamente elevada com as culturas de grãos (agricultores que dispõe de maiores superfícies) possuírem capacidade de investir, significativamente, na bovinocultura de leite. Isto condiciona a grande maioria dos produtores a desenvolver lentamente a atividade. Porém, tal processo não permite que estes agricultores acompanhem as exigências em qualidade e em quantidade que as indústrias estabelecem, após a produção ter atingido um nível que assegure o seu abastecimento.

$$
\text { BAPTISTA \& PORTELA (1995) }
$$

afirmam que as explorações familiares eminentemente diversificadas (produção de leite, batata, centeio, vinho e carne) e pouco especializadas permitem uma melhor valorização e racionalização dos recursos disponíveis, reduzindo os riscos e possibilitando uma reciclagem interna mais eficiente dos produtos e subprodutos. Esta característica de integração entre produções caracteriza uma enorme diversidade de UPFs, necessitando para sua compreensão ferramentas metodológicas apropriadas que, em parte, podem ser supridas pela abordagem sistêmica. Segundo SANTOS et al. (1994), quando se privilegia este enfoque, as pesquisas sócio-econômicas e agropecuárias buscam superar um modelo de adequação restrita, que analisa somente os elementos ou parte deles, enfocando os problemas de modo pontual. $\mathrm{Na}$ abordagem das explorações diversificadas e combinadas, procura-se potencializar a integração dos elementos em um conjunto maior. A pesquisa então se volta para o aprofundamento das relações sobre o produtor e sua família, a unidade de produção e o meio ambiente que os circunscreve.

A análise sistêmica tem, na tipologia, a forma de representar e compreender a heterogeneidade dos estabelecimentos agrícolas de uma dada região. Baseia-se nas práticas de funcionamento das propriedades, utilizando como atributos de classificação características estruturais peculiares ou sistemas praticados. É uma ferramenta que permite agrupar unidades de produção em tipos efetivamente comparáveis de unidades e possibilita, também, julgar o funcionamento do estabelecimento agrícola, detectando soluções possíveis aos problemas identificados, viabilizando a elaboração de recomendações técnicas adaptadas.

O objetivo principal deste trabalho é caracterizar, economicamente, os diferentes sistemas de produção praticados pelos produtores associados a COOPROL, visando subsidiar a determinação de ações diferenciadas para as distintas categorias de produtores.

\section{MATERIAL E MÉTODOS}

O trabalho foi desenvolvido na região da Depressão Central do RS, no período de janeiro a setembro de 1998. Apresentou como público alvo as UPFs associadas da COOPROL. As informações foram coletadas em um grupo de 53 propriedades, e foram obtidas com um instrumento denominado de "enquete" desenvolvido pelo grupo de pesquisa do Departamento de Ensino Agrícola e Extensão Rural da Universidade Federal de Santa Maria (DEAERUFSM). O diagnóstico das UPFs foi caracterizado por três momentos distintos e integrados: o estudo das condições estruturais das mesmas; o estudo do funcionamento das propriedades; o estudo da trajetória destas e dos objetivos do agricultor. Os critérios de agrupamento escolhidos estão associados àqueles fatores que diferenciam o processo de desenvolvimento das unidades: combinação das produções (variável predominante), características do ecossistema cultivado (condições de relevo), disponibilidade dos meios de produção, particularmente, da mão-de-obra, do tipo de força de tração (TA ou TM) e da SAU. Buscou-se apresentar uma imagem da realidade de cada sistema de produção, por meio de um procedimento que adota 
como referência à aglutinação das características em um modelo típico de UPF de cada sistema.

A determinação dos resultados econômicos das unidades, por sistema de produção, seguiu o modelo da determinação do Valor Agregado (VA) recomendado para a análise de UPF LIMA et al. (1995) e os elementos que fizeram parte da matriz do cálculo do VA, segundo a metodologia adotada, foram: Produto Bruto comercializado $(\mathrm{PBc})$, Consumo Intermediário (CI) e Depreciação (Dep). O VA é diferenciado em Valor Agregado Bruto comercializado (VABc) e Valor Agregado Líquido comercializado (VALc). Representados pelas seguintes equações: $\mathrm{VABc}=\mathrm{PBc}-\mathrm{CI}$; VALc $=\mathrm{PBc}-\mathrm{CI}-$ Dep.

Para a determinação da Renda Agrícola comercializada (RAc), subtraiu-se parte do VALc, que é repartido entre os vários agentes que intervêm no processo de produção, definido como a Distribuição do Valor Agregado (DVA). Obtém-se a RAc dando sequiência a mesma metodologia de cálculo, acrescentou-se a variável DVA na subtração do modelo matemático inicial: $\mathrm{RAc}=\mathrm{PBc}$ - CI Dep - DVA. Outros indicadores utilizados nesta análise são importantes na comparação entre e dentro das UPFs, que são representados pelas seguintes equações: Produtividade do Trabalho $(\mathrm{PW})=$ VALc/UTH $(\mathrm{UTH}=$ equivale à força de trabalho de um adulto, 17 a 59 anos, em 287 jornadas/ano); Remuneração monetária da Família $(\mathrm{RF})$ = RAc/UTH; desempenho por unidade de área $=\mathrm{VABc} / \mathrm{SAU}$.

Na comparação com a análise do nível de reprodução simples (NRS), procura-se responder em que medida o resultado do sistema de produção adotado pelo agricultor e sua família garante a reprodução das condições de força de trabalho ao longo do tempo. O ponto de partida na análise foi a modelagem do desempenho econômico do sistema e o modelo utilizado do VALc/UTH foi o descrito por LIMA et al. (1995). Este modelo é representado por uma equação linear do tipo $\mathrm{Y}=\mathrm{a} \mathrm{X}+\mathrm{b}$, no qual: "Y" representa o VALc; "a" que determina a inclinação da reta e representa o VABc por unidade de escala, que no caso é a SAU medida em "ha"; "X" representa a SAU explorada; "b" que é o ponto em que a reta atinge o eixo do $\mathrm{Y}$ (ordenada), representa a Dep total anual (b, que neste caso é negativo), medidas em reais.

\section{RESULTADOS E DISCUSSÃO}

Sistema de produção A - este sistema é composto por unidades que combinam a atividade de produção de leite com a cultura do arroz (Tabela 1) e apresentam restrições: na disponibilidade de SAU destinada à cultura do arroz, devido às condições ecológicas de encosta; o agricultor, na maioria das vezes, não possui terra própria; os equipamentos disponíveis são de uso manual e TA; a infraestrutura de instalação é composta por galpões de madeira; a atividade leiteira se caracteriza pela baixa escala de produção de leite comercializado/dia. O desempenho econômico do sistema se encontra no limite da viabilidade econômica, uma vez que possibilita a UPF atingir seus objetivos em NRS (NRS $=\mathrm{R} \$ 120,00$, salário mínimo regional no período, vezes 13 meses) (Figura 1), com uma área de $14,74 \mathrm{ha} / \mathrm{UTH}$ que se encontra próxima à disponibilidade máxima de SAU/UTH. Nesta condição, para LIMA et al. (1995), quanto mais eficiente e rápido o agricultor readequar o sistema, mais cedo a UPF ingressará no estágio de acumulação, destacando-se pelo resultado econômico da unidade (VAL/UTH) se situar acima do NRS (Figura 1). Portanto, no sistema há uma necessidade de elevar a produtividade da terra. Uma alternativa possível é buscar um maior retorno econômico proveniente da exploração leiteira, através de ajustes nos fatores ligados ao gerenciamento interno da unidade, para melhorar o nível de eficácia técnica da atividade. Como um exemplo, se a produtividade por vaca atingisse 9 1/vaca/dia (índice obtido por unidades em condições de produção semelhante), isto representaria um comércio diário de 50 litros e aumentaria a produtividade da terra de $\mathrm{R} \$ 119,38 /$ ha para $\mathrm{R} \$ 149,65 /$ ha de VABc/SAU.

Sistema de produção B - este sistema é composto por unidades que desenvolvem a atividade de produção de leite combinada com a cultura do arroz. Essas unidades localizam-se em área com maior dis ponibilidade de várzea para a cultura do arroz, quando comparado ao sistema A. O agricultor na maioria das vezes possui terra própria e complementa a área de cultivo com arrendamentos. Possui tração mecanizada composta por um trator com potência média de 70HP, mais implementos de cultivo convencional. A infra-estrutura de instalação é composta por dois galpões de madeira e a atividade leiteira se caracteriza, também, por um reduzido volume de leite comercializado diariamente. Apresenta uma situação privilegiada quanto ao desempenho econômico, uma vez que consegue remunerar a mão-de-obra familiar ao NRS, utilizando uma área de 10,74ha/UTH (Figura 1) e gera um $\mathrm{VABc} / \mathrm{SAU}$ de $\mathrm{R} \$ 253,85 /$ há. Isso permite que a mão-de-obra da UPF seja remunerada além do salário mínimo regional, caracterizando uma 
Tabela 1 - Caracterização dos sistemas de produção com bovinocultura de leite, observados no ano de 1997, segundo a disponibilidade de mão-de-obra, superfície agrícola útil, escala de produção e recursos utilizados no processo de produtivo, situados na região da Depressão Central do RS.

\begin{tabular}{|c|c|c|c|c|c|c|c|c|c|}
\hline \multirow[b]{2}{*}{ Variáveis } & \multicolumn{9}{|c|}{ Sistemas de produção } \\
\hline & A & $\mathrm{B}$ & $\mathrm{C}$ & $\mathrm{D}$ & $\mathrm{E}$ & $\mathrm{F}$ & G & $\mathrm{H}$ & I \\
\hline \multirow{2}{*}{$\begin{array}{l}\text { Mão-de-obra disponível (UTH) } \\
\text { Superfície agrícola útil (SAU) em hectares }\end{array}$} & 2 & 3 & 2,5 & 3,5 & 2,5 & 3,5 & 2,5 & 2,5 & 2,5 \\
\hline & 30 & 54 & 20 & 50 & 40 & 308 & 80 & 20 & 45 \\
\hline Arroz (Oryza sativa) $*$ & 6 & 22 & - & - & - & 2 & - & - & - \\
\hline E $\quad$ Milho (Zea mays) $*$ & - & 14,5 & 2 & 10 & - & - & - & - & - \\
\hline ‡ै Mandioca (Manihot esculenta)* & - & - & 1 & 1,5 & - & - & - & - & - \\
\hline E Batata-doce (Ipomoea batatas)* & - & - & 1 & 1,0 & - & - & - & - & - \\
\hline $\bar{\Xi} \approx$ Melancia (Citrulus lonatus)* & - & - & 1 & - & - & - & - & - & - \\
\hline ‡ี ప్ Moranga (Curcubita máxima)* & - & - & 0,35 & - & - & - & - & - & - \\
\hline ¿ Feijão-preto (Plaseolus vulgaris)* & - & - & - & - & 1,5 & 2 & - & - & - \\
\hline E Sorgo (fabricação de vassoura)* & - & - & - & - & 0,5 & 7 & - & - & - \\
\hline$\exists \quad$ Forrageiras cultivadas & - & - & 2 & 3,5 & 1,5 & 5 & 10,5 & 3 & 7 \\
\hline Subsistência & 7,5 & 1,5 & - & - & 5,0 & 20 & 12 & 4 & 4,5 \\
\hline$\ddot{A}$ Campo nativo & 16,5 & 16 & $\begin{array}{c}12,6 \\
5\end{array}$ & 34 & 31,5 & 272 & 57,5 & 13 & 33,5 \\
\hline \multirow{3}{*}{$\begin{array}{l}\text { Leite comercializado/dia (litros) } \\
\text { Produtividade/vaca /dia (litros) } \\
\text { Depreciação da UPF/UTH** }\end{array}$} & 15 & 30 & 80 & 110 & 16 & 42 & 36 & 130 & 120 \\
\hline & 4,0 & 4,6 & 8,8 & 9,0 & 2,52 & 2,6 & 6,0 & 13,5 & 7,7 \\
\hline & 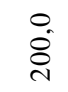 & $\hat{o}_{0}^{0}$ & $\begin{array}{l}0 \\
0 \\
n \\
n\end{array}$ & $\begin{array}{l}0 \\
\stackrel{0}{0} \\
i\end{array}$ & $\stackrel{0}{\stackrel{0}{0}}$ & $\stackrel{0}{0}$ & $\begin{array}{l}0 \\
\infty \\
\infty \\
\dot{\infty}\end{array}$ & @̊. & $\stackrel{\circ}{\overparen{a}}$ \\
\hline \multirow{4}{*}{$\begin{array}{l}\text { Valor agregado } \\
\text { Líquido UTH/SAU }\end{array}$} & $\stackrel{\Xi}{\Xi}$ & I & $\stackrel{\widetilde{I}}{\tilde{I}}$ & $\frac{\pi}{\pi}$ & $\stackrel{\pi}{=}$ & $\stackrel{\pi}{=}$ & $\stackrel{\pi}{=}$ & $\Xi$ & $\Xi$ \\
\hline & $\stackrel{n}{=}$ & $\stackrel{\infty}{=}$ & $\infty$ & \pm & 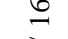 & $\infty$ & $\approx$ & $\infty$ & $\stackrel{\infty}{-}$ \\
\hline & $\overline{0}$ & $\overline{0}$ & $\partial$ & $\bar{\theta}$ & ले & $\gamma_{\text {. }}$ & 于 & $\vec{F}_{0}$ & $\bar{a}$ \\
\hline & ஜ & ֻ̊ & $\begin{array}{l}\dot{0} \\
\infty \\
\infty\end{array}$ & $\begin{array}{l}\infty \\
\stackrel{\infty}{I}\end{array}$ & 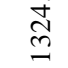 & $\frac{\nabla}{7}$ & in & $\underline{2}$ & $\stackrel{1}{\Sigma}$ \\
\hline
\end{tabular}

*Culturas desenvolvidas com fim comercial;

** A depreciação aparece como um valor negativo, pois, embora não ocorrendo produção agrícola, ela existe.

Obs.: As variáveis se encontram relacionadas nas colunas.

situação de reprodução ampliada. Segundo LIMA et al. (1995), nestas condições é possível a realização de investimentos nas atividades produtivas, melhorias no padrão de vida da família e eventual aumento na superfície agrícola.

Sistema de produção $\mathrm{C}$ - este sistema é composto por unidades que desenvolvem a atividade de produção de leite combinada com hortigranjeiros e apresenta como peculiaridades: baixa variação na demanda da mão-de-obra durante o ano agrícola, resultado da comercialização de produtos in natura diretamente ao consumidor; localiza-se em áreas de coxilhas; o produtor geralmente possui parte da SAU; os equipamentos disponíveis são de uso manual e TA; infra-estrutura de instalação é similar ao sistema $\mathrm{A}$; a atividade leiteira se distingue por apresentar uma escala de produção superior aos sistemas A e B (Tabela 1), sendo parte desta produção entregue à Cooperativa e outra comercializada in natura ou terceirizada. $\mathrm{O}$ desempenho econômico do sistema é coerente com o NRS, pois possibilita que a UPF atinja seus objetivos, com uma área de 6,94ha/UTH (Figura 1). A produtividade da terra é expressa por um $\mathrm{VABc} / \mathrm{SAU}$ de $\mathrm{R} \$ 301,25 / \mathrm{ha}$, retratando que este modelo apresenta uma eficiência técnica superior aos sistemas A e B, principalmente, na escala de produção do leite e na renda extra, gerada pelo comércio in natura, do leite e dos hortigranjeiros. Neste sentido comprova-se a observação realizada por ESCOSTEGUY et al. (1993), que as "miudezas" em uma UPF como: leite, mandioca, batata-doce, abóbora entre outras, são responsáveis por boa parte da reprodução das UPFs.

Sistema de produção D - é formado por unidades que desenvolvem a atividade leiteira combinada com hortigranjeiros. Essas unidades localizam-se em áreas com condições topográficas similares ao sistema $\mathrm{C}$ e o produtor geralmente tem a posse de quase toda SAU. Os equipamentos disponíveis de uso manual, de TA e de TM, como: um trator com potência de $65 \mathrm{CV}$; implementos de cultivo convencional e de plantio direto; ensiladeiras, estas em sociedade com UPFs vizinhas. A infra-estrutura de instalação não apresenta particularidades e a atividade leiteira é caracterizada por uma escala mais elevada de produção de leite comercializado. $\mathrm{O}$ desempenho econômico do sistema não atinge o NRS, com uma disponibilidade de área de 14,29ha/UTH (Figura 1). A produtividade 


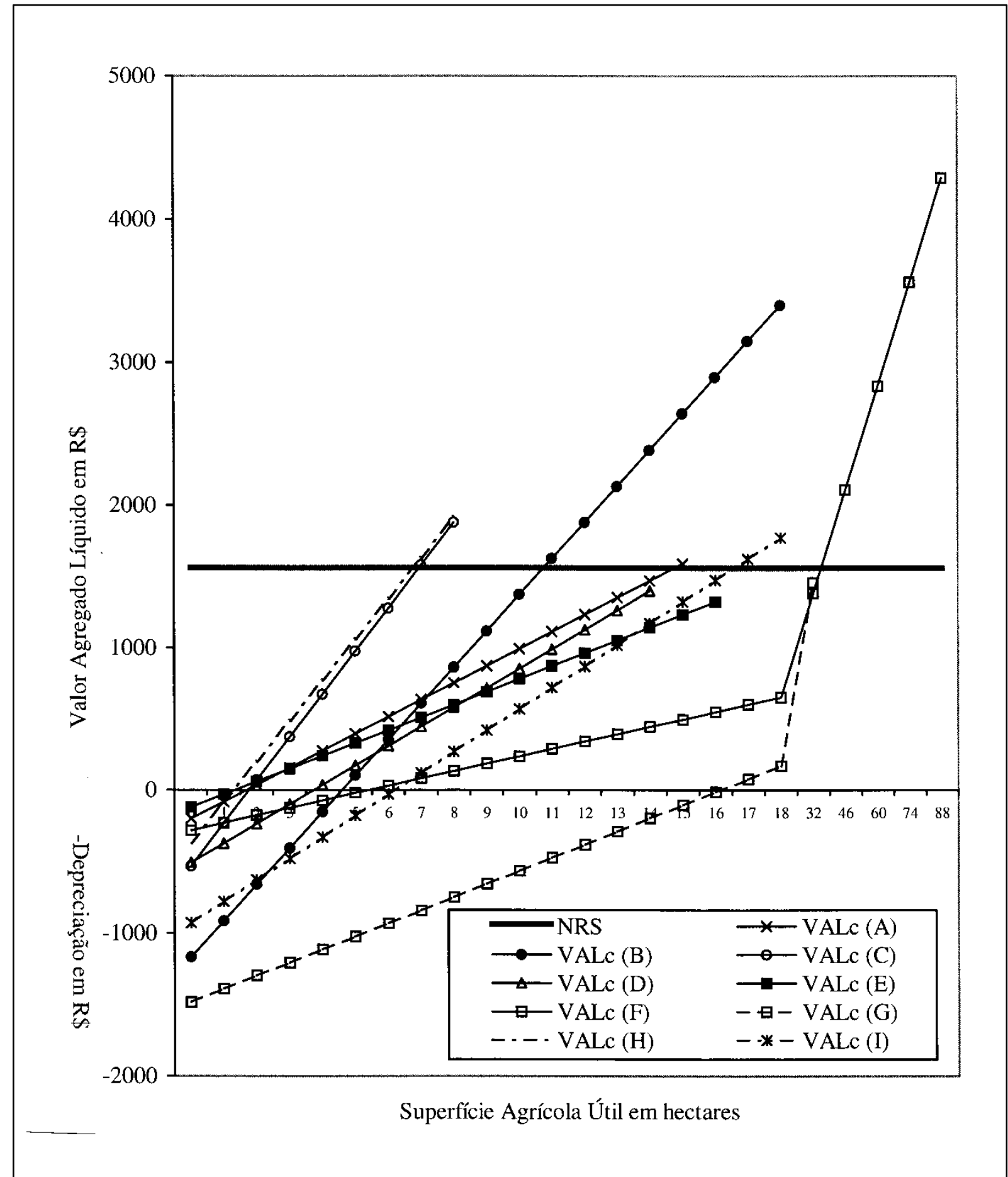

Figura 1 - Representação do desempenho econômico dos modelos de UPFs, pertencente aos sistemas de produção com bovinocultura de leite, quanto ao Valor Agregado Líquido comercializado (VALc) partindo da Depreciação, Nível de Reprodução Simples (NRS), por UTH em relação à disponibilidade de SAU/UTH, 1997.

da terra em termos de $\mathrm{VABc} / \mathrm{SAU}$ é de $\mathrm{R} \$ 136,10 /$ ha, retratando que o modelo de produção não é eficaz tecnicamente quanto à disponibilidade dos recursos existentes na unidade. A perspectiva do sistema é de reorientação para que as unidades atinjam o NRS. Considerando-se a mesma estrutura de produção, seria necessário gerar R \$ 151,61/ha de $\mathrm{VABc} / \mathrm{SAU}$, possível de ser atingido a partir dos 156

Ciência Rural, v. 32, n. 5, 2002. 
litros de leite comercializados/dia, que desencadearia um processo inicial de capitalização nas unidades. As propriedades que se enquadram neste sistema necessitam, num primeiro momento, traçar metas de desempenho técnico (número de vacas lactantes/SAU, produtividade por vaca, etc.), que possam responder a um resultado econômico superior ao NRS.

Sistema de produção E- este sistema é composto por unidades que desenvolvem a atividade leiteira combinada com bovinos de corte. Localizam-se em áreas com relevo íngreme (montanhoso), e o produtor, geralmente, possui somente parte da SAL; os equipamentos disponíveis são de uso manual e com TA. A infra-estrutura de instalação é similar aos sistemas anteriores e a atividade leiteira apresenta uma escala de produção similar ao sistema A. O desempenho econômico apresentado não possibilita que a UPF atinja o NRS (Figura 1), com uma disponibilidade de área de 16ha/UTH, o que caracteriza um processo contínuo de descapitalização. A produtividade da terra em termos de $\mathrm{VABc} / \mathrm{SAU}$ é de $\mathrm{R} \$ 90,27 / \mathrm{ha}$, retratando que o modelo de produção não é tecnicamente adequado. Há uma perspectiva que o sistema, caso não aconteça uma mudança, sofra um processo de exclusão em curto prazo. Para reverter este quadro, deverão ser adotadas algumas mudanças em relação aos fatores de produção, considerando a atual escala de produtividade do leite. Para que a unidade consiga atingir o NRS, com a mesma estrutura de produção, é preciso gerar $\mathrm{R} \$ 113,39 / \mathrm{ha}$ de VAB/SAU. Neste sistema existe a necessidade de preceder a inversão de características genéticas de parte do rebanho, buscando uma maior aptidão leiteira. Portanto, com 60 litros de leite comercializados/dia, a unidade remunerará a mãode-obra acima do salário mínimo regional.

Sistema de produção $\mathrm{F}$ - este sistema é composto por UPFs que praticam a combinação de atividades de produção de leite com bovinos de corte e agricultura de encosta. $\mathrm{O}$ produtor possui quase a totalidade da SAU, a restante é arrendada de terceiros. Os equipamentos disponíveis são de uso manual e de TA e o leite é produzido em pequena escala. $\mathrm{O}$ desempenho econômico do sistema possibilita que a UPF atinja o NRS, com uma área disponível de 32,54ha/UTH (Figura 1). A produtividade da terra em termos de $\mathrm{VABc} / \mathrm{SAU}$ é baixa $\mathrm{R} \$ 51,93 / \mathrm{ha}$, retratando que o modelo não é tecnicamente compatível quando comparado com outros sistemas. Portanto, os níveis de desempenho destas áreas somente compensam, economicamente, porque as UPFs se encontram em uma situação privilegiada quanto à disponibilidade de terra (Tabela 1).
Sistema de produção $\mathrm{G}$ - este sistema é desenvolvido por unidades que praticam a combinação das atividades de produção de leite com bovinos de corte com tração mecanizada. Localizase geralmente em áreas de coxilha e tem origem com a divisão de antigas fazendas. O leite é produzido em escala reduzida. $\mathrm{O}$ desempenho econômico do sistema não permite que a UPF atinja o NRS (Figura 1), com uma área disponível de 32ha/UTH. A produtividade da terra em termos de $\mathrm{VABc} / \mathrm{SAU}$ é baixa, em média $\mathrm{R} \$ 91,88 / \mathrm{ha}$, retratando que o modelo de produção não é economicamente compatível com as disponibilidades dos recursos. Uma alternativa para a UPF é o aumento na participação das atividades vegetais, ou mesmo, uma reorientação da produção animal para um maior ingresso por parte da atividade leiteira. Neste contexto, considerando a RAc/UTH para que a propriedade possa ser sustentável, a atividade leiteira deve atingir no mínimo uma produção de 105 litros de leite comercializados/dia mantendo os demais condicionantes estáveis.

Sistema de produção $\mathrm{H}$ - este sistema é composto por unidades que desenvolvem a atividade leiteira como única fonte de renda. Localiza-se em áreas que apresentam formações de encosta/coxilha. $\mathrm{O}$ produtor geralmente possui a terra e supre a demanda por SAU arrendando. Os equipamentos disponíveis são de uso manual e TA. Entre os sistemas é o que apresenta o maior volume de leite comercializado diariamente. $\mathrm{O}$ desempenho econômico possibilita que a UPF atinja os objetivos quanto ao NRS, com uma área de 6,76ha/UTH (Figura 1). A produtividade da terra é expressa pelo $\mathrm{VABc} / \mathrm{SAU}$ de $\mathrm{R} \$ 285,53 / \mathrm{ha}$, retratando que o modelo de produção expõe uma eficiência técnica procedente de uma boa produtividade por vaca, o que gera uma maior escala de leite comercializado, quando comparado com os sistemas anteriores. A perspectiva para o sistema é que continue em um processo lento de capitalização à medida que mantém inalteradas as condições de produção e de mercado.

Sistema de produção I - neste sistema, a atividade de produção de leite aparece como única fonte geradora de renda, similar ao sistema anterior. Localiza-se em áreas que apresentam condições naturais similares ao sistema $\mathrm{H}, \quad$ o produtor normalmente possui a maior parte da SAU. As máquinas e equipamentos disponíveis são de uso manual, TA e TM, similares aos sistemas anteriores que desenvolvem agricultura na coxilha. A atividade leiteira se distingue pela cadeia de venda do leite, que está representada pelas unidades que, embora, integradas a Cooperativa, vendem parte da produção 
in natura. A coerência do sistema se resume em atender os objetivos quanto ao NRS, obtido com uma área de 16,58ha/UTH (Figura 1). A produtividade da terra expressa pelo $\mathrm{VABc} / \mathrm{SAU}$ de $\mathrm{R} \$ 150,12 /$ ha, retrata um modelo de produção, em relação à eficácia técnica, inferior ao sistema $\mathrm{H}$, condição, que resulta de uma menor produtividade/vaca.

\section{CONCLUSÕES}

Em relação aos sistemas $\mathrm{D}, \mathrm{E}$ e $\mathrm{G}$, a sua viabilidade em NRS, com a mesma estrutura de produção, pode ser obtida aumentando a participação da atividade de produção de leite, em diferentes graus, conforme as particularidades de cada sistema. As propostas de intervenção para os sistemas A e I devem redimensionar os recursos disponíveis, internos às UPFs, para um maior volume na comercialização do leite.

As performances das atividades vegetais no sistema B e C, embora diferenciadas, conduzem junto com a eficiência, nas transformações internas que ocorrem nestes sistemas, a uma alta concentração do $\mathrm{VABc} / \mathrm{SAU}$.

$\mathrm{O}$ sistema $\mathrm{F}$, embora apresente uma baixa produtividade da terra, tem um resultado final economicamente compensador, devido à privilegiada disponibilidade de terra. No sistema $\mathrm{H}$, a posição em nível de reprodução simples procede da elevada produtividade da terra.

A atual situação de produção de leite, de um modo geral, conduz a uma diferenciação sócioeconômica mais acentuada entre os agricultores que apresentam maior dependência econômica desta atividade. Os que conseguem produzir mais no inverno, recebem uma remuneração maior pelo produto no decorrer do ano, o que possibilita reinvestirem na atividade. Em outra situação, os produtores em descapitalização tendem a limitar-se à manutenção das condições com que vêm sobrevivendo.

\section{REFERÊNCIAS BIBLIOGRÁFICAS}

BAPTISTA, A., PORTELA, J. Pastagens temporárias ou forrageiras anuais para as pequenas explorações de leite nos vales Sub-Montanos de Trás-os-Montes. Revista Portuguesa de Zootecnia, ano II, n.1, p.1-21, 1995.

ESCOSTEGUY, C, DAMBORIARENA, E, FREITAS, P. A produção de leite como alternativa para os pequenos agricultores: relato de uma atividade de campo. Ijuí: UNIJUI, 1993. 72p.

LIMA, A.P., BASSO, N., NEUMANN, P.S., $\boldsymbol{e}$ t $\boldsymbol{a l}$. Administração da unidade familiar: modalidades de trabalho com agricultores. Ijui, RS : UNIJUI, 1995. 175p.

NETO, B S. BASO, D e LIMA, A, P. Sistemas de produção da região de Três de Maio (RS): 1) História agrária e diferenciação social dos agricultores. In: ENCONTRO DA SOCIEDADE BRASILEIRA DE SISTEMAS DE PRODUÇÃO, 3, 1998, Florianópolis. Anais... Florianópolis SC : SBS, 1998. CD ROOM.

SANTOS, Z.A.P.S., SOUZA, M.C.M., CARRIERI, A.P. Pesquisa em sistemas de produção. Revista Agricultura, São Paulo, v.41, n.2, p.127-139, 1994.

TESTA, V.M., NADAL, R., MIOR, L.C., et al. O desenvolvimento sustentável do Oeste Catarinense: proposta para discussão. Florianópolis : EPAGRI, 1996. 247p. 